4. Deligne, P., Mumford, D.: The irreducibility of the space of curves of a given genus. Publ. math. I.H.E.S. 36, 75-110 (1969)

5. Faltings, G.: Calculus on arithmetic surfaces. Eingereicht bei Ann. of Math.

6. Faltings, G.: Arakelov's theorem for abelian varieties. Invent. math. 73, 337-347 (1983)

7. Moret-Bailly, L.: Variétés abéliennes polarisées sur les corps de fonctions. C.R. Acad. Sc. Paris 296, 267-270 (1983)

8. Namikawa, Y.: Toroidal compactification of Siegel spaces. Lecture Notes in Mathematics, vol. 812. Berlin-Heidelberg-New York: Springer 1980

9. Parshin, A.N.: Algebraic curves over function fields I. Math. USSR Izvestija 2, 1145-1170 (1968)

10. Raynaud, M.: Schémas en groupes de type $(p, \ldots, p)$. Bull. Soc. Math. France 102, 241-280 (1974)

11. Szpiro, L.: Sur le théorème de rigidité de Parsin et Arakelov. Astérisque 64, 169-202 (1979)

12. Szpiro, L.: Séminaire sur les pinceaux de courbes de genre au moins deux. Astérisque 86 (1981)

13. Tate, J.: $p$-divisible groups. Proceedings of a conference on local fields, Driebergen 1966, pp. 158-183. Berlin-Heidelberg-New York: Springer 1967

14. Tate, J.: Endomorphisms of abelian varieties over finite fields. Invent. math. 2, 134-144 (1966)

15. Zarhin, J.G.: Isogenies of abelian varieties over fields of finite characteristics. Math. USSR Sbornik 24, 451-461 (1974)

16. Zarhin, J.G.: A remark on endomorphisms of abelian varieties over function fields of finite characteristics. Math. USSR Izvestija 8, 477-480 (1974)

Oblatum 8-VI \& 10-VII-1983

\title{
Zusatz bei der Korrektur
}

Herr $\mathrm{O}$. Gabber hat mir mitgeteilt, daß der Beweis von Satz 2 nicht ganz korrekt ist. Man erhält nur, daß die Folge $h\left(A_{n}\right)$ stationär wird. Dies reicht für unsere Zwecke. 